\title{
Effect of rosuvastatin on the expression of candidate gene GALNT3 in atherosclerosis
}

\author{
YANSHAO LIU, TAO CHEN and JIEYONG XING \\ Department of Cardiovascular Medicine, Jinan Zhangqiu District Hospital of \\ Traditional Chinese Medicine, Jinan, Shandong 250200, P.R. China
}

Received August 21, 2017; Accepted February 1, 2018

DOI: $10.3892 / \mathrm{etm} .2018 .6008$

\begin{abstract}
The effect of rosuvastatin on the expression of candidate gene polypeptide $\mathrm{N}$-acetylgalactosaminyltrans ferase 3 (GALNT3) in atherosclerosis was studied. Sixty Wistar rats were randomly divided into the control $(n=20)$, model (atherosclerosis group, $\mathrm{n}=20$ ) and administration (rosuvastatin group, $\mathrm{n}=20$ ) groups. The atherosclerosis model was established via injecting D3.6 million units of vitamin per kilogram of body weight and then the rats were fed with high-fat diet for 6 weeks. The total cholesterol, serum triglyceride and nitric oxide contents were detected using the kits, the morphological changes in thoracic aorta were observed via hematoxylin and eosin (H\&E) staining, the mRNA expression of candidate gene GALNT3 was detected via reverse transcription-polymerase chain reaction (RT-PCR), and the protein expression of candidate gene GALNT3 was detected via western blot analysis. Compared with those in the control group, the contents of serum triglyceride and total cholesterol in the model group were significantly increased, and then significantly decreased after drug administration. Morphological observation showed that the surface of thoracic aorta was not smooth with endothelial shedding, and the smooth muscle cells were arranged irregularly and their number was obviously increased. Moreover, RT-PCR and western blot analysis revealed that the mRNA and protein expressions of GALNT3 were significantly increased in the administration group. Rosuvastatin can therefore significantly upregulate the expression of candidate gene GALNT3 in atherosclerosis, thereby reducing the incidence of atherosclerosis.
\end{abstract}

Correspondence to: Dr Jieyong Xing, Department of Cardiovascular Medicine, Jinan Zhangqiu District Hospital of Traditional Chinese Medicine, 1463 Embroidered Water Street, Mingshui Street Office, Jinan, Shandong 250200, P.R. China

E-mail: xingjieyongjn@126.com

Key words: rosuvastatin, atherosclerosis, candidate gene polypeptide $\mathrm{N}$-acetylgalactosaminyltransferase 3

\section{Introduction}

The incidence of atherosclerosis (AS) has been on the increase in recent years. As is a chronic inflammatory disease of blood vessels, which poses a great threat to human health (1-4). The process from the hyperlipidemia at first to AS involves the accumulation of lipid on the arterial intima, occurrence of fibrous lesions and formation of atherosclerotic plaques, resulting in vascular stenosis, decreased elasticity and increased thickness of vascular wall, ultimately causing other serious complications, such as thrombotic disease, cerebrovascular disease and coronary heart disease. The pathogenesis is the aggregation of alarge number of cytokines, macrophage-derived foam cells and a variety of chemokines on the intimal surface, causing endothelial cell lesions and ultimately leading to the occurrence of AS (5). Vascular endothelial cell damage is a key factor in inducing atherosclerotic disease. Under physiological conditions, vascular endothelial cells can prevent the accumulation of inflammatory factors on the vascular wall, and reduce the pathological migration and proliferation of smooth muscle cells, ultimately improving the occurrence and development of the disease (6). At present, the progression mechanism of atherosclerotic disease is not fully clarified, but the inflammatory response of vascular endothelial cells caused by intravascular lipid accumulation and metabolic disorders is closely related to the occurrence and development of AS $(7,8)$.

Statins, such as the hydroxymethylglutaryl coenzyme A reductase inhibitor, can effectively suppress the synthesis of cholesterol and reduce the serum cholesterol content (9). Rosuvastatin is a new type of statin, which, not only reduces the content of blood lipids, but also improves the physiological effect of vascular endothelium (10). However, there is little research on its effect on the expression of candidate gene polypeptide $\mathrm{N}$-acetylgalactosaminyltransferase 3 (GALNT3). This study aimed to investigate the effect of rosuvastatin on the expression of AS candidate gene GALNT3, to lay a foundation for the clinical treatment of AS with rosuvastatin.

\section{Materials and methods}

Materials and reagents. Rosuvastatin (5 mg/tablet; Lunan Beite Pharmaceutical Co., Ltd., Linyi, China); nitric oxide (NO), triglyceride (TG) and total cholesterol (TC) detection kits (Nanjing Jiancheng Biotechnology Institute, Nanjing, 
China); rabbit anti-rat GALNT3 polyclonal antibody and goat anti-rabbit horseradish peroxidase (HRP)-labeled secondary polyclonal antibody (cat. nos. 16716-1-AP and SA00001-2; Proteintech; Wuhan Institute of Biological Products Co., Lts., Wuhan, China); GALNT3 antibody and horseradish peroxidase (HRP)-labeled secondary antibody (Proteintech; Wuhan Institute of Biological Products Co., Lts.); primer synthesis, reverse transcription kit and quantitative PCR kit (Takara, Dalian, China); bicinchoninic acid (BCA) protein quantification kit and cell lysis buffer (Beyotime Biotechnology Research Institute, Nantong, China) were used in this study.

Experimental animals and grouping. Sixty clean Wistar rats weighing $180 \pm 10 \mathrm{~g}$, were randomly divided into the control $(n=20)$, model (AS group, $n=20)$ and administration (rosuvastatin group, $n=20$ ) groups. The rats were injected with D3.6 million units of vitamin per kilogram of body weight first, and then fed with high-fat diet $(87.8 \%$ basal feed, $10 \%$ lard oil, $2 \%$ cholesterol) for 6 weeks. The rats in the administration group were fed with high-fat diet every day and treated with gavage using $1 \mathrm{mg} / \mathrm{kg}$ rosuvastatin calcium at the same time. The study was approved by the Ethics Committee of Jinan Zhangqiu District Hospital of Traditional Chinese Medicine (Jinan, China).

Detection of TC, TG and NO levels. After modeling, the blood was taken from the orbital cavity of rats under anesthesia using the capillary tube. After coagulation, the blood was centrifuged at $8,000 \mathrm{x} \mathrm{g}$ for $10 \mathrm{~min}$, the upper serum was taken and the serum TG, TC and NO levels were detected according to the protocol of the kit.

Morphological observation of vascular tissues. After modeling, the vascular tissues were taken from the thoracic aorta of rats, and immediately fixed with $4 \%$ paraformaldehyde solution for $48 \mathrm{~h}$, followed by dehydration and transparency, paraffin embedding, cutting and sticking, dewaxing and staining, sealing, and preparation into hematoxylin and eosin (H\&E) staining sections. The effect of rosuvastatin on the morphology of vascular tissues was observed under a microscope (Olympus Corporation, Tokyo, Japan).

Detection of mRNA expression of GALNT3 via RT-PCR. The vascular tissues were taken from the thoracic aorta of rats, and the total RNA was extracted. The qualified total RNA was used as the template to synthesize cDNA via reverse transcription. The specific reaction conditions were as follows: Incubation at $42^{\circ} \mathrm{C}$ for $15 \mathrm{~min}$, incubation at $95^{\circ} \mathrm{C}$ for $3 \mathrm{~min}$, cooling on ice and stored at $-80^{\circ} \mathrm{C}$ for subsequent experiment. Routine amplification was performed according to the primer sequence in Table I.

Western blot analysis. The vascular tissues were taken from the thoracic aorta of rats, and the total protein was extracted. Then the protein concentration was determined and the samples to be tested were treated. Protein $(50 \mu \mathrm{g})$ was taken for sodium dodecyl sulfate polyacrylamide gel electrophoresis (SDS-PAGE) and transferred onto the membrane. The isolated protein was electronically transferred onto the polyvinylidene fluoride (PVDF) membrane. The membrane
Table I. RT-PCR primer sequences of GALNT3 mRNA.

\begin{tabular}{ll}
\hline Gene name & \multicolumn{1}{c}{ Primer sequences } \\
\hline GALNT3 & F: 5'-GTTGCTAGGAGCAACAGTCGCA-3' \\
& R: 5'-AGTTCACCGTGGTAGTATTGTAGT-3' \\
GAPDH & F: 5'-CTCAACTACATGGTCTACATG-3' \\
& R: 5'-TGGCATGGACTGTGGTCATGAG-3'
\end{tabular}

RT-PCR, reverse transcription-polymerase chain reaction; GALNT3, polypeptide $\mathrm{N}$-acetylgalactosaminyltransferase 3; GAPDH, glyceraldehyde 3-phosphate dehydrogenase.

was sealed using blocking solution at room temperature for $1 \mathrm{~h}$, followed by incubation with primary antibody overnight at $4^{\circ} \mathrm{C}$. After the membrane was washed with TTBS, the secondary antibody $(1: 2,000)$ was added for incubation at room temperature for $1 \mathrm{~h}$. The membrane was washed again with TTBS, followed by color development using developing solution and photographed.

Statistical analysis. Data were expressed as mean \pm standard deviation and processed using SPSS 17.0 (SPSS, Inc., Chicago, IL, USA). One-way analysis of variance (ANOVA) and the SNK post hoc test were used for the statistical analysis of data obtained. $\mathrm{P}<0.05$ was considered to indicate a statistically significant difference.

\section{Results}

Effect of rosuvastatin on blood lipids in AS rats. As shown in Fig. 1, the contents of TG and TC in the model group were significantly higher than those in the control group at 6 weeks after modeling, suggesting that the early AS model was successfully established. After administration of rosuvastatin, the contents of TG and TC in serum were significantly decreased $(\mathrm{P}<0.01)$.

Effect of rosuvastatin on the vascular morphology of rats. As shown in Fig. 2, the results of H\&E staining showed that in the control group, the vascular intima was smooth, medial smooth muscle cells and elastic fibers were arranged regularly without obvious proliferation. In the model group, the vascular surface was not smooth with endothelial shedding and irregular arrangement, the nuclear staining was deep, and the intercellular matrix and thickness of vascular wall were increased.

Effect of rosuvastatin on the mRNA expression of GALNT3. As shown in Fig. 3, the mRNA expression level of GALNT3 in model group was significantly increased compared with that in the control group $(\mathrm{P}<0.01)$. By contrast, the mRNA expression level of GALNT3 in the administration group was significantly decreased $(\mathrm{P}<0.01)$, indicating that rosuvastatin can significantly reduce the mRNA expression of GALNT3 in AS rats.

Effect of rosuvastatin on the protein expression of GALNT3. As shown in Fig. 4, the protein expression level of GALNT3 
A

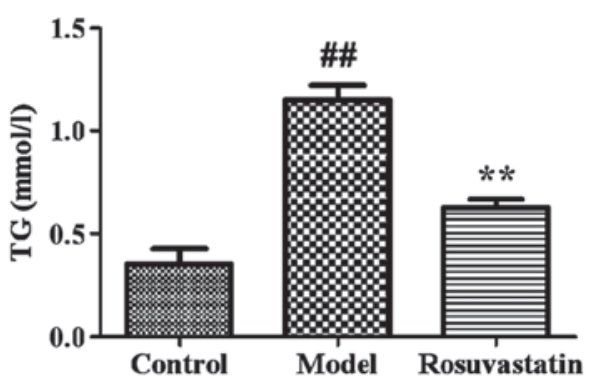

B

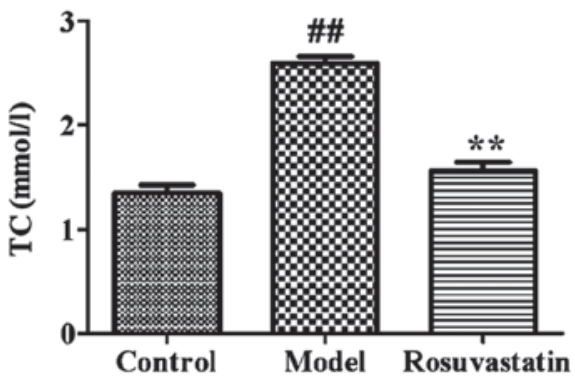

C

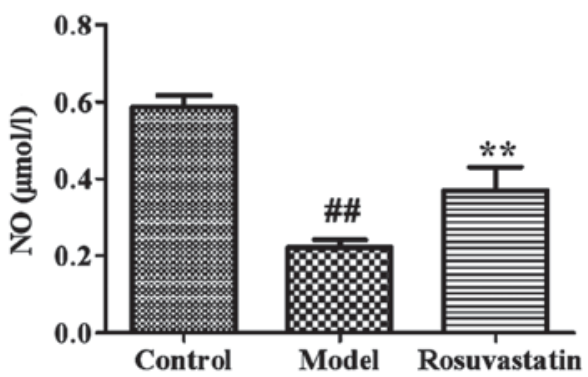

Figure 1. Effect of rosuvastatin on (A) serum triglyceride (TG), (B) total cholesterol (TC) and (C) nitric oxide (NO) contents; ${ }^{\# \#} \mathrm{P}<0.01$ compared with control group; ${ }^{* *} \mathrm{P}<0.01$ compared with model group.
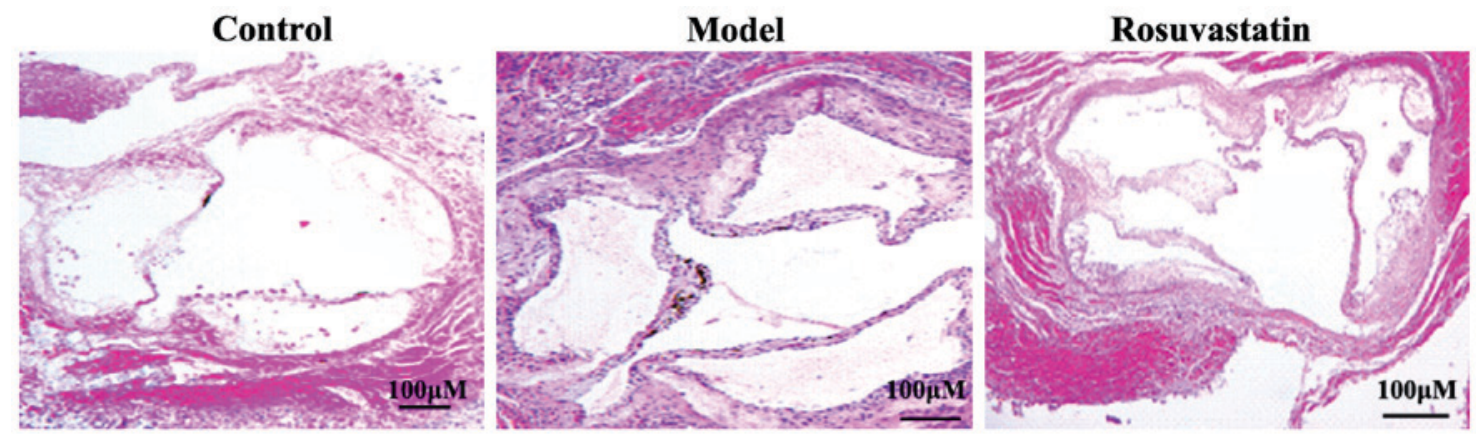

Figure 2. Effect of rosuvastatin on polypeptide N-acetylgalactosaminyltransferase 3 (GALNT3) mRNA via RT-PCR. RT-PCR, reverse transcription-polymerase chain reaction.

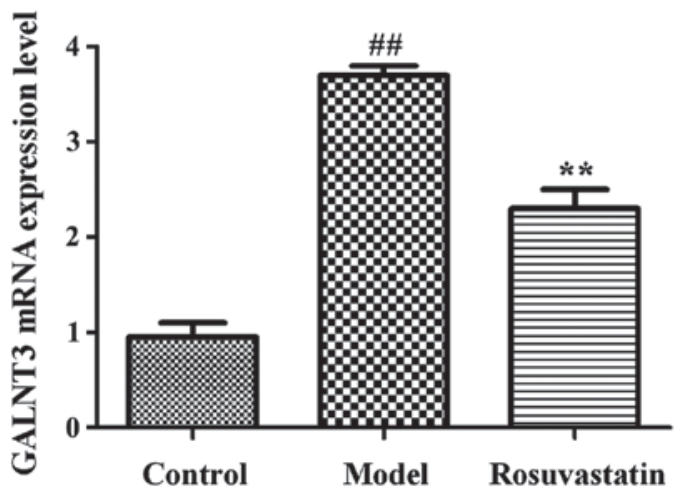

Figure 3. Effect of rosuvastatin on polypeptide $\mathrm{N}$-acetylgalactosaminyl-trans ferase 3 (GALNT3) mRNA via RT-PCR; ${ }^{\# \#} \mathrm{P}<0.01$ compared with control group; ${ }^{* *} \mathrm{P}<0.01$ compared with model group.

in the model group was significantly decreased compared with that in the control group, and the protein expression level of GALNT3 in the administration group was also significantly decreased $(\mathrm{P}<0.01)$, indicating that rosuvastatin can significantly downregulate the protein expression of GALNT3 in AS.

\section{Discussion}

The complex pathogenesis of AS is due to the combined effects of external and genetic factors, which involves a variety of genes and other factors. Although some achievements have been made in the study on AS, there is still a lack of effective
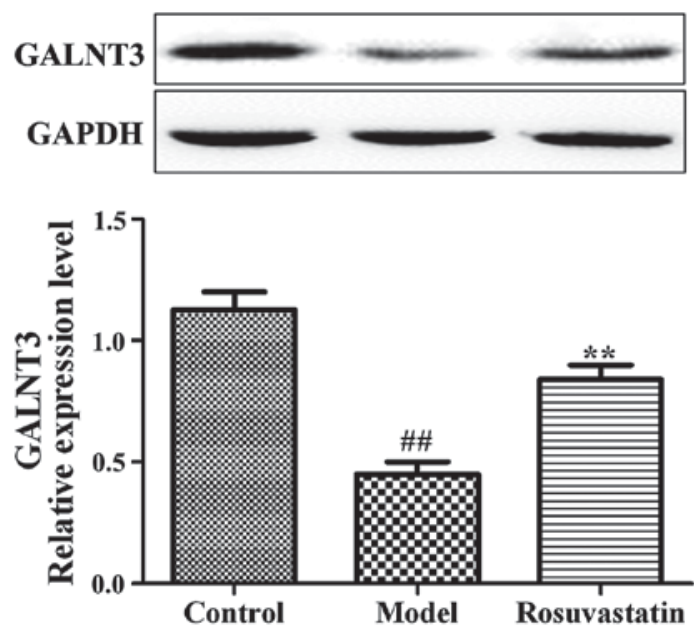

Figure 4. Effect of rosuvastatin on polypeptide $\mathrm{N}$-acetylgalactosaminyl-trans ferase 3 (GALNT3) protein via western blot analysis; ${ }^{\# \#} \mathrm{P}<0.01$ compared with control group; ${ }^{* *} \mathrm{P}<0.01$ compared with model group.

treatment means. Therefore, finding an effective therapeutic target and mechanism of AS is of groundbreaking medical significance for the clinical treatment and intervention of AS.

Vascular endothelium can secrete a variety of bioactive substances, among which NO derived from endothelium has a strong cardiovascular protection and anti-AS effect. NO is a kind of gas signaling molecule produced by vascular endothelium under the action of endothelial nitric oxide synthase (eNOS). $\mathrm{NO}$, not only has a strong vasodilatory effect, but can also inhibit platelet aggregation and adhesion, prevent thrombosis, inhibit 
the expression of inflammatory factors, low-density lipoprotein oxidation and foam cell formation, and prevent the occurrence of early AS. In addition, NO can inhibit the proliferation and migration of vascular smooth muscle cells, and protect and prevent progression in the late stage of AS. Therefore, NO is an essential protective factor that resists AS and thrombosis, and maintains the normal vasomotor response in cardiovascular system. Clinical and experimental data show that glycoprotein is a key factor that maintains the steady state of endothelial cells and blood vessels, which is also an important signal regulator of cardiovascular function (11). In sugar complexes, the role of glycoprotein is very significant, which, not only includes the regulation of cell growth and differentiation, but also plays an important role in the identification, signal transduction and immune response, carrying and transmitting a large number of biological information (12). The O-linked glycoprotein is mainly distributed on the cell surface, and the catalytic response of O-glycosylation reaction is involved in the formation of this important skeleton. The members of UDP-GalNAc are the performers and participants in this biochemical process, and the expression of each member in the ppGalNAc-Ts family has a certain periodic functional specificity, and many members, such as the rate-limiting enzymes, participate in the process of post-translational processing of a variety of proteins, which have irreplaceable physiological activities $(13,14)$. Additionally, GALNT3 gene has an important role in protecting vascular endothelial cells, and the damage to vascular endothelial cells is considered an important factor in the incidence of AS (15). Shibao et al reported that compared with that in normal breast tissues, the ppGALNT3 expression level in the differentiated malignant adenocarcinoma is significantly increased, and it is not expressed in normal breast tissues, but in breast cancer cells (16). In addition, the study on GALNT3 gene showed that the levels of vitamin $\mathrm{D}$ and $\mathrm{Ca}^{2+}$ can affect the expression of ppGALNT3 (17). However, there are few reports on the relationship between GALNT3 gene and coronary heart disease. It was found in this study to the best of our knowledge for the first time that rosuvastatin has a significant effect on the expression level of ppGALNT3 gene in AS, providing a new perspective for the treatment of AS.

In this study, the AS rat model was established using high-fat diet. The contents of serum TG, TC and NO in AS rats after intervention with rosuvastatin were investigated in this study. Compared with those in model group, the contents of TG and TC were significantly decreased after drug administration, but the serum NO content was significantly increased. The results of H\&E staining showed that in model group, the vascular intima of thoracic aorta was not smooth with endothelial shedding, and the smooth muscle cells were arranged irregularly with proliferation; but the conditions were significantly improved after the administration of rosuvastatin. The results of RT-PCR and western blot analysis revealed that compared with those in model group, the mRNA and protein expressions of GALNT3 were significantly increased in the administration group. Similar research showed that the proliferation of vascular endothelial cells can be significantly inhibited through intervention in the GALNT3 expression in human umbilical vein endothelial cells (HUVECs), while the proliferation of vascular endothelial cells is significantly increased and the arterial plaques are obviously reduced after the upregulation of GALNT3 $(18,19)$. In addition, findings have also shown that consistent results are observed in the detection of vascular endothelial cell apoptosis using a flow cytometer. In other words, the endothelial cell apoptosis is aggravated when the GALNT3 gene expression is inhibited, but the upregulation of GALNT3 can significantly inhibit apoptosis of endothelial cells and alleviate AS $(20,21)$. In conclusion, rosuvastatin can significantly increase the expression of AS candidate gene GALNT3, thereby improving atherosclerotic lesions.

\section{Acknowledgements}

Not applicable.

\section{Funding}

No funding was received.

\section{Availability of data and materials}

All data generated or analyzed during this study are included in this published article.

\section{Authors' contributions}

YL contributed significantly to writing the manuscript and cell culture. TC analyzed and interpreted western blot analysis. JX recorded and analysed orthological observation of vascular tissues and RT-PCR. All authors read and approved the final manuscript.

\section{Ethics approval and consent to participate}

The study was approved by the Ethics Committee of Jinan Zhangqiu District Hospital of Traditional Chinese Medicine (Jinan, China).

\section{Consent for publication}

Not applicable.

\section{Competing interests}

The authors declare that they have no competing interests.

\section{References}

1. van Gils JM, Ramkhelawon B, Fernandes L, Stewart MC, Guo L, Seibert T, Menezes GB, Cara DC, Chow C, Kinane TB, et al: Endothelial expression of guidance cues in vessel wall homeostasis dysregulation under proatherosclerotic conditions. Arterioscler Thromb Vasc Biol 33: 911-919, 2013.

2. Kriszbacher I, Koppán M and Bódis J: Inflammation, atherosclerosis, and coronary artery disease. N Engl J Med 353: 429-430, 2005.

3. Ross R: Atherosclerosis - an inflammatory disease. N Engl J Med 340: 115-126, 1999.

4. Gregersen I, Holm S, Dahl TB, Halvorsen B and Aukrust P: A focus on inflammation as a major risk factor for atherosclerotic cardiovascular diseases. Expert Rev Cardiovasc Ther 14: 391-403, 2016.

5. Barton M: Obesity and aging: Determinants of endothelial cell dysfunction and atherosclerosis. Pflugers Arch 460: 825-837, 2010 . 
6. Gotlieb AI: Smooth muscle and endothelial cell function in the pathogenesis of atherosclerosis. Can Med Assoc J 126: 903-908, 1982.

7. Ross R and Glomset JA: The pathogenesis of atherosclerosis (first of two parts). N Engl J Med 295: 369-377, 1976.

8. Ross R and Glomset JA: The pathogenesis of atherosclerosis (second of two parts). N Engl J Med 295: 420-425, 1976.

9. Vale N, Nordmann AJ, Schwartz GG, de Lemos J, Colivicchi F den Hartog F, Ostadal P, Macin SM, Liem AH, Mills E, et al: Statins for acute coronary syndrome. Cochrane Database Syst Rev 9: CD006870, 2011.

10. Durán WN, Breslin JW and Sánchez FA: The NO cascade, eNOS location, and microvascular permeability. Cardiovasc Res 87: 254-261, 2010.

11. Tian XY, Wong WT, Xu A, Chen ZY, Lu Y, Liu LM, Lee VW Lau CW, Yao X and Huang Y: Rosuvastatin improves endothelial function in $\mathrm{db} / \mathrm{db}$ mice: Role of angiotensin II type 1 receptors and oxidative stress. Br J Pharmacol 164: 598-606, 2011.

12. Tsuiji H, Takasaki S, Sakamoto M, Irimura T and Hirohashi S: Aberrant O-glycosylation inhibits stable expression of dysadherin, a carcinoma-associated antigen, and facilitates cell-cell adhesion. Glycobiology 13: 521-527, 2003.

13. SørensenT, WhiteT,WandallHH,Kristensen AK, RoepstorffP and Clausen H: UDP-N-acetyl-alpha-D-galactosamine:polypeptide $\mathrm{N}$-acetylgalactosaminyltransferase. Identification and separation of two distinct transferase activities. J Biol Chem 270: 24166-24173, 1995.

14. Ten Hagen KG, Fritz TA and Tabak LA: All in the family: The UDP-GalNAc:polypeptide N-acetylgalactosaminyltransferases. Glycobiology 13: 1R-16R, 2003.

15. Vita JA and Keaney JF Jr: Endothelial function: A barometer for cardiovascular risk? Circulation 106: 640-642, 2002.

16. Shibao K, Izumi H, Nakayama Y, Ohta R, Nagata N, Nomoto M Matsuo K, Yamada Y, Kitazato K, Itoh H, et al: Expression of UDP-N-acetyl-alpha-D-galactosamine-polypeptide galNAc $\mathrm{N}$-acetylgalactosaminyl transferase- 3 in relation to differentiation and prognosis in patients with colorectal carcinoma. Cancer 94: 1939-1946, 2002.
17. Chefetz I, Kohno K, Izumi H, Uitto J, Richard G and Sprecher E: GALNT3, a gene associated with hyperphosphatemic familial tumoral calcinosis, is transcriptionally regulated by extracellular phosphate and modulates matrix metalloproteinase activity. Biochim Biophys Acta 1792: 61-67, 2009.

18. Ramakrishnan P,Clark PM,Mason DE,Peters EC,Hsieh-Wilson LC and Baltimore D: Activation of the transcriptional function of the NF- $\kappa$ B protein $c-R e l$ by O-GlcNAc glycosylation. Sci Signal 6: ra75, 2013.

19. Gill DJ, Tham KM, Chia J, Wang SC, Steentoft C, Clausen H, Bard-Chapeau EA and Bard FA: Initiation of GalNAc-type $\mathrm{O}$-glycosylation in the endoplasmic reticulum promotes cancer cell invasiveness. Proc Natl Acad Sci USA 110: E3152-E3161, 2013.

20. Bakhashab S, Lary S, Ahmed F, Schulten HJ, Bashir A, Ahmed FW, Al-Malki AL, Jamal HS, Gari MA and Weaver JU: Reference genes for expression studies in hypoxia and hyperglycemia models in human umbilical vein endothelial cells. G3 (Bethesda) 4: 2159-2165, 2014.

21. Saffi MA, Furtado MV, Polanczyk CA, Montenegro MM, Ribeiro IW, Kampits C, Haas AN, Rösing CK and Rabelo-Silva ER: Relationship between vascular endothelium and periodontal disease in atherosclerotic lesions: Review article. World J Cardiol 7: 26-30, 2015

(i) () () This work is licensed under a Creative Commons

CY Attribution-NonCommercial-NoDerivatives 4.0 International (CC BY-NC-ND 4.0) License. 